\title{
COMPORTAMIENTO DE LA MORTALIDAD EN EL HOSPITAL INFANTIL DR. ROBERT REID CABRAL, SANTO DOMINGO, REPÚBLICA DOMINICANA, PERIODO 2013-2017
}

\section{Mortality behavior at Dr. Robert Reid Cabral Children's Hospital, Santo Domingo, Dominican Republic, 2013-2017}

\section{Ricardo Elías Melgena, Cristina Contreras ${ }^{\mathrm{b}}$ y Cristian Díaz Rodríguez}

Recibido: 7 de agosto, 2019 • Aprobado: 12 de octubre, 2019

Cómo citar: Elías Melgen R, Contreras C, Díaz Rodríguez C. Comportamiento de la mortalidad en el hospital infantil Dr. Robert Reid Cabral, Santo Domingo, República Dominicana, período 2013-2017. cysa [Internet]. 3 de marzo de 2020 [citado 4 de marzo de 2020];4(1):49-5. Disponible en: https://revistas.intec.edu.do/index.php/cisa/article/view/1667

\section{Resumen}

Introducción: el análisis de la mortalidad hospitalaria permite evaluar la calidad de la asistencia médica. Las últimas publicaciones sobre mortalidad en el Hospital Infantil Dr. Robert Reid Cabral datan de 1997.

Objetivo: conocer el comportamiento de la mortalidad en el Hospital Infantil Dr. Robert Reid Cabral durante el periodo 2013-2017

Métodos: estudio descriptivo. Se revisaron los libros de registro y actas de defunciones de los pacientes fallecidos en el Hospital Dr. Robert Reid Cabral durante el periodo 2013-2017.

Resultados: 3,800 pacientes fallecieron, en promedio 760 fallecidos por año (margen 679-868). $79.9 \%$ con menos de 5 ańos de edad; las muertes en menores de 1 ańo de edad representaron el $61.3 \%$ y en el periodo neonatal el $26.1 \%$. La Sepsis/Shock Séptico (43.4\%) y la Hemorragia Pulmonar (15.4\%) fueron los dos principales diagnósticos de defunción; el $34 \%$ de los fallecidos presentaban una morbilidad asociada al diagnóstico de defunción y en el $40 \%$ la morbilidad asociada era una malformación congénita. El 69.5 \% fallecieron en la Unidad de Cuidados Intensivos Pediátricos o Neonatal y $40 \%$ de los pacientes fallecieron antes de las 48 horas de su ingreso al hospital.

a Departamento de Neumología, Hospital Infantil Dr. Robert Reid Cabral, Centro Nacional de Investigación en Salud Materno Infantil Dr. Hugo Mendoza (CENISMI), República Dominicana

Correo-e: reliasmelgen@yahoo,es

\begin{abstract}
Introduction: the analysis of hospital mortality allows to evaluate the quality of medical care. The latest publications on mortality at Dr. Robert Reid Cabral Children's Hospital date from 1997.
\end{abstract}

Objective: to know the behavior of mortality in the Dr. Robert Reid Cabral Children's Hospital during the period 2013-2017

Methods: descriptive study. Registration books and death certificates of deceased patients at Dr. Robert Reid Cabral Hospital during 2013-2017 were reviewed.

Results: 3,800 patients died, on average 760 deaths per year (margin 679-868). $79.9 \%$ with less than 5 years of age; deaths in children under 1 year of age represented $61.3 \%$ and in the neonatal period $26.1 \%$. Sepsis / Septic Shock (43.4\%) and Pulmonary Hemorrhage (15.4\%) were the two main diagnoses of death; $34 \%$ of the deceased had a morbidity associated with the diagnosis of death and in $40 \%$ the associated morbidity was a congenital malformation. $69.5 \%$ died in the Pediatric or Neonatal Intensive Care Unit and $40 \%$ of the patients died within 48 hours of admission to the hospital.

\footnotetext{
b Departamento de Urgencia Hospital Infantil Dr. Robert Reid Cabral, República Dominicana. Correo-e: crismi117@hotmail.com

c Residencia de Pediatría, Hospital Infantil Dr. Robert Reid Cabral, República Dominicana. Correo-e: doctordiaz73@gmail.com
} 
Conclusión: el grupo etario de mayor mortalidad son los menores de 1 año de edad, se evidencia un alto porcentaje de muertes con enfermedad crónica y malformaciones congénitas asociadas. El hecho de que el $40 \%$ de los pacientes fallecieran antes de las 48 horas de su ingreso, indica la condición de gravedad en que estos pacientes llegan al hospital.

Palabras clave: mortalidad infantil; causa de muerte; factor de riesgo; República Dominicana; malformación congénita.

\section{Introducción}

La mortalidad en los diferentes grupos etarios ha presentado cambios importantes, por una transición epidemiológica de la que la población infantil no escapa; esta variación en las causas de mortalidad se viene produciendo de acuerdo al nivel de desarrollo de cada país. ${ }^{1-3}$

La mortalidad infantil es considerada como un indicador clave del nivel de salud de una población, y es usado para medir la efectividad del sistema de atención de salud de un país y su grado de desarrollo. ${ }^{4}$ El medio socioeconómico en que el niño nace y se desarrolla influye decisivamente sobre los riesgos de muerte. La pobreza, el bajo nivel educativo y la falta de servicios básicos, tornan al niño más vulnerable, impidiendo el acceso y restándole eficacia a la tecnología médica disponible, para prevenir o tratar la mayoría de las enfermedades infantiles. ${ }^{5}$

Las principales causas de muerte infantil han cambiado radicalmente en las últimas décadas; el componente neonatal, los accidentes, cardiopatías, neoplasias y enfermedades congénitas o malformaciones han dejado a las causas infecciosas al final de la lista de las diez principales de muerte infantil. ${ }^{6,7}$

Gautier $^{8}$ encontró que entre las primeras diez causas de las 1,620 defunciones ocurridas durante el año 1990 en el Hospital Infantil Dr. Robert Reid Cabral estaban: sepsis (38.3\%), gastroenteritis (11.9\%),
Conclusion: the age group with the highest mortality are those under 1 year of age, a high percentage of deaths with chronic disease and associated congenital malformations are evident. The fact that $40 \%$ of patients died before 48 hours of admission indicates the serious condition in which these patients arrive at the hospital.

Keywords: Child mortality; death cause; risk factor; Dominican Republic; congenital malformation.

neumonía $(10.8 \%)$, meningitis $(8.3 \%)$, prematuridad $(3.3 \%)$, cardiopatías congénitas (2.9\%), quemaduras $(2.8 \%)$, anemias $(2.0 \%)$, anomalías congénitas gastrointestinales $(1.9 \%)$ y encefalitis (1.4\%); igualmente Perez Then ${ }^{9}$ en el año 1997 encontró, entre las principales causas de muertes, las enfermedades infectocontagiosas, destacándose la enfermedad diarreica aguda y la infección de vías respiratorias.

Conocer la mortalidad hospitalaria ha permitido evaluar la calidad de la asistencia y detectar resultados adversos. La calidad asistencial basada en el análisis sistemático de la mortalidad y en los posibles efectos adversos relacionados con el fallecimiento se ha estudiado con el objetivo de elaborar propuestas de mejora. ${ }^{10}$

El comportamiento de la mortalidad en el Hospital Infantil Dr. Robert Reid Cabral, hospital de tercer nivel de atención y referencia nacional en pediatría, ha sido estudiada por décadas, pero las últimas publicaciones datan de 1997. El objetivo de la presente investigación es conocer el comportamiento de la mortalidad en el Hospital Infantil Dr. Robert Reid Cabral durante el periodo 2013 - 2017.

\section{Material y método}

Se trata de un estudio descriptivo, cuya información se obtuvo considerando todos los fallecidos durante el periodo 2013-2017 registrados en el Departamento de Estadística del Hospital Dr. Robert Reid 
Cabral, para ello se revisaron los libros de registro de defunciones y las actas de defunciones. Las variables de estudio se recolectaron en un instrumento diseñado para estos fines y los datos se procesaron en una base de datos diseñada en Excel; para el análisis se usó estadística descriptiva. Toda información recolectada se utilizó bajo estricta confidencialidad por parte de los investigadores, y no se dio a conocer datos pertinentes de los pacientes y sus familiares.

\section{Resultados}

Durante el periodo de estudio, año 2013 al 2017, en el Hospital Infantil Dr. Robert Reid Cabral se registraron 3,800 fallecimientos, un promedio de 760 fallecidos por año (margen 679-868).

El $79.9 \%$ de los pacientes fallecidos tenían menos de 5 años de edad, aquellos con menos de 1 año de edad representaron el $61.3 \%$ y de estos, el $26.1 \%$ tenían menos de 28 días de vida (periodo neonatal). (Tabla 1)

Tabla 1. Distribución por edad al momento de fallecer

\begin{tabular}{|c|c|c|c|}
\hline Edad & $\mathbf{N}$ & $\mathbf{\%}$ & $\begin{array}{c}\% \\
\text { Acumulado }\end{array}$ \\
\hline 0-28 días (neonatal) & 991 & 26.1 & 26.1 \\
\hline 29 días - 1 año & 1,336 & 35.2 & 61.3 \\
\hline 1 a 5 años & 708 & 18.6 & 79.9 \\
\hline mayor de 5 años & 765 & 20.1 & 100 \\
\hline Total & $\mathbf{3 , 8 0 0}$ & & \\
\hline
\end{tabular}

Fuente: elaboración propia a partir de los libros de registro y actas de defunciones HIRRC
El $55.4 \%(2,104)$ de los fallecidos eran del sexo masculino; de acuerdo al lugar de residencia, el mayor porcentaje, $52.0 \%(1,975)$, residían en la Región de Salud Metropolitana, seguidos por los que residían en la Región de Salud de Valdesia, $19.2 \%$ (730) y $12.1 \%$ (459) en la Región de salud Este y $0.3 \%$ (11) procedían de Haití; la tabla 2 muestra el lugar de residencia de estos pacientes.

Tabla 2. Lugar de residencia del fallecido

\begin{tabular}{|l|c|c|}
\hline \multicolumn{1}{|c|}{ Lugar de residencia } & N & \% \\
\hline Región Metropolitana & 1,975 & 52.0 \\
\hline Región Valdesia & 730 & 19.2 \\
\hline Región Este & 459 & 12.1 \\
\hline Región el Valle & 243 & 6.4 \\
\hline Región Enriquillo & 198 & 5.2 \\
\hline Región Nordeste & 75 & 2.0 \\
\hline Región Cibao Central & 69 & 1.8 \\
\hline Región Norcentral & 14 & 0.4 \\
\hline Región Cibao Occidental & 8 & 0.2 \\
\hline Haití & 11 & 0.3 \\
\hline No registrado & 18 & 0.5 \\
\hline & $\mathbf{3 8 0 0}$ & \\
\hline \multicolumn{1}{|c|}{ Total } & &
\end{tabular}

Fuente: elaboración propia a partir de los libros de registro y actas de defunciones HIRRC

El $40.3 \%(1,533)$ de los pacientes, falleció antes de las 48 horas de haber ingresado al hospital. El área en donde estos pacientes estaban hospitalizados al momento de fallecer era: Unidad de Cuidados Intensivos Pediátricos (40.5\%), Unidad de Recién Nacidos (29.0\%), Sala de Hospitalización (11.9\%), Área de Urgencias (9.9\%) y Área de Emergencias (8.6\%). (Tabla 3). 
Tabla 3. Área del hospital donde fallecieron los pacientes

\begin{tabular}{|c|c|c|}
\hline Área del hospital & $\mathbf{N}$ & $\%$ \\
\hline UCIP & 1,540 & 40.5 \\
\hline Recién nacidos & 1,103 & 29.0 \\
\hline Hospitalización & 454 & 11.9 \\
\hline Urgencias & 375 & 9.9 \\
\hline Emergencias & 328 & 8.6 \\
\hline Total & 3,800 & \\
\hline
\end{tabular}

Fuente: elaboración propia a partir de los libros de registro y actas de defunciones HIRRC

La tabla 4, muestra los 10 principales diagnósticos de defunción durante el periodo de estudio, siendo la sepsis y el shock séptico $(43.4 \%)$ y la hemorragia pulmonar (15.4\%) las dos primeras causas.

Tabla 4. Principales diagnósticos de defunción

\begin{tabular}{|l|c|}
\hline \multicolumn{1}{|c|}{ Diagnóstico de defunción } & $\%$ \\
\hline Sepsis - Shock Séptico & 43.4 \\
\hline Hemorragia Pulmonar & 15.4 \\
\hline Hemorragia e hipertensión intracraneal & 6.3 \\
\hline $\begin{array}{l}\text { Shock Cardiogénico e Insuficiencia } \\
\text { Cardíaca }\end{array}$ & 6.2 \\
\hline Insuficiencia respiratoria & 5.4 \\
\hline Sangrado gastrointestinal & 4.8 \\
\hline Fallo multiorgánico & 4.1 \\
\hline $\begin{array}{l}\text { Shock Hipovolémico con desequilibrio } \\
\text { hidroelectrolítico }\end{array}$ & 3.8 \\
\hline Coagulación intravascular diseminada & 2.5 \\
\hline Otros diagnósticos de defunción & 8.1 \\
\hline
\end{tabular}

Fuente: elaboración propia a partir de los libros de registro y actas de defunciones HIRRC
El 34 \% $(1,292)$ de los fallecidos presentaban una morbilidad asociada al diagnóstico de defunción; la principal morbilidad asociada, en el $40 \%$ (517) de los casos, era una malformación congénita y el $56.3 \%$ (291) de estas malformaciones congénitas eran cardiopatías congénitas y malformaciones del Sistema Nervioso Central con Hidrocefalias, $24.7 \%$ (128). La tabla 5 muestra las otras comorbilidades que padecían estos pacientes al momento de fallecer.

Tabla 5. Comorbilidades asociadas

\begin{tabular}{|c|c|c|}
\hline Comorbilidad & N & \% \\
\hline Malformación congénita & 517 & 40.0 \\
\hline Desnutrición & 215 & 16.6 \\
\hline Prematuridad & 153 & 11.8 \\
\hline $\begin{array}{c}\text { Síndrome genético y } \\
\text { retraso psicomotor }\end{array}$ & 149 & 11.5 \\
\hline $\begin{array}{c}\text { Patología Hematológica } \\
\text { Inmunocompromiso }\end{array}$ & 141 & 10.9 \\
\hline $\begin{array}{c}\text { Insuficiencia Renal Crónica } \\
\text { Patología Pulmonar }\end{array}$ & 37 & 24.3 \\
\hline \begin{tabular}{c} 
Total comorbilidad \\
\hline
\end{tabular} & $\mathbf{1 , 2 9 2}$ & 1.9 \\
\hline
\end{tabular}

Fuente: elaboración propia a partir de los libros de registro $\mathrm{y}$ actas de defunciones HIRRC 


\section{Comportamiento de la mortalidad en el Hospital Infantil Dr. Robert Reid Cabral, Santo Domingo, República Dominicana, periodo 2013-2017}

\section{Discusión}

En la mortalidad infantil influyen distintos factores: la edad de los niños, las condiciones socioeconómicas de cómo viven y la inversión en salud. En aquellos menores de 27 días de vida, prevalecen los determinantes vinculados a los servicios de salud (atención del embarazo, parto y del neonato) y en la salud de los más grandes (entre 28 y 365 días de vida), tienen mayor influencia las condiciones ambientales y socioeconómicas en las que crece y se desarrolla el niño. ${ }^{11}$ Se ha demostrado, como una disminución del gasto sanitario público se asocia con un aumento de la mortalidad infantil, siendo mayor este incremento en los países de bajos ingresos. ${ }^{12}$

La probabilidad de morir antes de los 5 años de edad en República Dominicana, para el año 2016, era de 31 por cada 1,000 nacidos vivos, una de las tasas más altas de la Región de Las Américas; el $84 \%$ de estas muertes corresponden a niños fallecidos con menos de 1 año de vida, y el $68 \%$ corresponden a muertes neonatales, menos de 28 días de vida. ${ }^{13}$ Por lo general, las muertes por causas exógenas (enfermedades infectocontagiosa) obedecen a un control de los factores del ambiente, de la inmunización masiva de la población y al uso de nuevas drogas, como los antibióticos; cobrando así progresiva importancia otros grupos de causas, entre ellas, las de origen endógeno, es decir, las malformaciones congénitas y las originadas en el período perinatal. ${ }^{14}$

En esta revisión se ha podido evidenciar, que al igual de lo encontrado en la década de los 90, el grupo etario que presenta mayor mortalidad es en los menores de 1 ańo de edad con $62 \%$; por otro lado Gautier y Prez-Then expusieron que el mayor porcentaje de los fallecidos correspondían a causas exógenas, reportándose sobre el $70 \%$ del total de las muertes, por causas infectocontagiosas ${ }^{8,9} \mathrm{y}$ a pesar de que en la actualidad la sepsis y el schok séptico representaron la primera causa directa de muerte, un alto porcentaje de fallecidos (34 \%) tenía una enfermedad crónica asociada; siendo las malformaciones congénitas la más frecuente, como son las cardiopatías congénitas y malformaciones del SNC con hidrocefalia, lo que condiciona mayor morbi mortalidad en estos pacientes.

En México, entre 1980 y 2005, la tasa de mortalidad infantil descendió de 40,7 a 16,9 por 1000 nacimientos; en cambio, la tasa de mortalidad específica por malformaciones congénitas creció de 2,2 a 3,5 por 1000 nacimientos; aquellas con buen pronóstico y tratamiento programable en unidades con equipamiento básico, fueron las únicas que mostraron una tendencia descendente, mientras que las que requieren tratamiento urgente en unidades especializadas mostraron tasas crecientes. ${ }^{15}$

El mayor porcentaje de pacientes fallecidos (83\%) residían en las zonas de mayor cercanía al hospital (Regiones de Salud Metropolitana, Valdesia y el Este). Llama la atención que en el $40.3 \%$ de los fallecidos, la defunción ocurrió antes de las 48 horas de haber ingresado al hospital, lo que refleja las condiciones de gravedad en las que fueron admitidos estos pacientes, generalmente por demora en buscar atención o condiciones inadecuadas en el traslado; el $18.5 \%$ fallecieron en las áreas de urgencia/emergencia del hospital, con pocas horas de estadía.

El comportamiento de la mortalidad en el Hospital Infantil Dr. Robert Reid Cabral, durante el periodo estudiado, refleja una situación similar de otros hospitales pediátricos de la región, ${ }^{4,16,17}$ alta mortalidad en menores de 1 años y una combinación entre causas exógenas y endógenas en la estructura de causas de muertes. Se debe considerar, que una atención oportuna garantizando adecuadas condiciones en el traslado de los pacientes, contribuirá a disminuir la mortalidad y favorecerá una mayor tasa de sobrevivencia. 


\section{Bibliografía}

1. Omran AR. The epidemiologic transition. A theory of the epidemiology of population change. Milbank Mem Fund Q 1971;49(4): 509-38. Available from: https://www.ncbi.nlm.nih.gov/ pmc/articles/PMC2690264/

2. Wang H, Dwyer-Lindgren L, Lofgren KT, Rajaratnam JK, et al. Age-specific and sex-specific mortality in 187 countries, 1970-2010: a systematic analysis for the Global Burden of Disease Study 2010. Lancet 2012;380(9859): 2071-94. Available from: https://www.ncbi.nlm.nih.gov/ pubmed/23245603

3. Behm Rosas H. Determinantes económicosy sociales de la mortalidad en América Latina. Revista Cubana de Salud Pública. 2017;43(2). Available from: http://scielo.sld.cu/scielo.php?script=sci_ arttext\&pid=S0864-34662017000200013

4. Segredo Molina Y. La mortalidad hospitalaria en el Hospital Pediátrico "José Luis Miranda" Rev Cubana Pediatr. 2017; 89(4). Available from: http://www.revpediatria.sld.cu/index.php/ped/ article/view/209/152

5. Ana M. Kaempffer R; Ernesto Medina L. Análisis de la mortalidad infantil y factores condicionantes. Chile 1998 Rev. chil. pediatr. v.71 n.5 Santiago set. 2000. Available from https://scielo. conicyt.cl/scielo.php?script=sci_arttext\&pid= S0370-41062000000500005.

6. Alonso E, Villán J. Mortalidad infantil en un hospital de nivel terciario. Limitación de esfuerzo terapéutico, correspondencia clínico-patológica y precisión diagnóstica. Anales de pediatría, Asociación Española de Pediatría. 2012 junio;76(6). Available from: https://www.analesdepediatria. org/es-mortalidad-infantil-un-hospital-nivel-articulo-S1695403311005042\#bib0005
7. Barría-Pailaquilén RM y col. Tendencia de la mortalidad infantil y de neonatos menores de 32 semanas y de muy bajo peso. Rev. Latino-Am. Enfermagem jul.-ago. 2011;19(4) Available from: http://www.scielo.br/pdf/rlae/v19n4/es_17.pdf

8. Gautier-Abréu T, y col. Mortalidad infantil durante el año 1990 en la Clínica Infantil Dr. Robert Reid Cabral. Acta Méd Dom. 1992; 14(l): 1-3.

9. Pérez E. Morbi-mortalidad en la Clínica infantil Dr. Robert Reid Cabral. Arch Dom Ped DRISSN-fiXX-0606 ADOERBIO M2 enero-abril 1997;33(I).

10. Romaguera Monzonís A et al. Análisis de la mortalidad como herramienta de mejora de la calidad asistencial en un servicio de cirugía general Cir Esp. 2006;80(2): 78-82. Available from https:// www.elsevier.es/es-revista-cirugia-espanola-36-pdf-13091091

11. Finkelsteina JZ, Duhaua M, Speranzac A, Marconib E, Escobar P. Evolución de la mortalidad infantil en Argentina en el marco de los Objetivos de Desarrollo del Milenio. Arch Argent Pediatr 2016;114(3): 216-22. Available from: https://www.sap.org.ar/docs/publicaciones/ archivosarg/2016/v114n3a05.pdf

12. Esperanza Olcina MJ, González Rodríguez MP. Disminuir el gasto público sanitario aumenta la mortalidad infantil. Evid Pediatr. 2015;11:59. Available from: http://archivos.evidenciasenpediatria.es/DetalleArticulo/_LLP3k9qgzIh7aNQ BiadwmQ4oeNia7MhT0WsdY1dUdyL1wCs 5bbTOCJY_55ctTElvuSiGCN_W_Gp0EXzh mIpHcg

13. UNICEF. Estado Mundial de la Infancia 2017. [Online] Available from: https://www.unicef. org/spanish/publications/files/SOWC_2017_ SP.pdf [Accessed 18th September 2018]. 
14. Mazzeo V. La mortalidad infantil en Argentina. Revista Población y Desarrollo: Argonautas y Caminantes, 2014;10. Available from: file:/// C:/Users/Rafael\%20J\%202/Downloads/ 1734-6198-1-PB.pdf

15. Gómez-Alcalá A, Rascón-Pacheco R. La mortalidad infantil por malformaciones congénitas en México: un problema de oportunidad y acceso al tratamiento Rev Panam Salud Públical Pan Am J Public Health. 2008;24(5). Available from: https://www.scielosp.org/pdf/rpsp/2008. v24n5/297-303/es

16. Ferrari M, Ferreira A, De Leonardis D, Fernández A, Imbriaco J. Mortalidad hospitalaria en un hospital pediátrico de referencia nacional: Centro Hospitalario Pereira Rossell. Rev Med Uruguay 2002;18: 59-65 Available from: http://www. scielo.edu.uy/pdf/rmu/v18n1/v18n1a07.pdf
17. Díaz-Garrido D, Pinto-Zaldumbide SC, Lazo-Álvarez MA, Cárdenas-Vivero AS, Valencia-Calderón CJ, Vásquez-Hahn C et al. Causas de defunción en un hospital pediátrico de tercer nivel de atención en Ecuador. Rev Mex Pediatr 2018; 85(6): 207-11. Available from: https:// www.medigraphic.com/pdfs/pediat/sp-2018/ sp186c.pdf 